Uday Khopkar · Roger M. Williams · Peter Torrie

\title{
Prehysterectomy embolisation of uterine arteries for large uterine fibroids-a report
}

Received: 1 October 2004 / Accepted: 4 January 2005/Published online: 15 March 2005

(C) Springer-Verlag Berlin / Heidelberg 2005

\begin{abstract}
Hysterectomy for large uterine fibroids can prove to be a technically difficult procedure. The irregular shape of the uterus filling the pelvis may make access to the pedicles difficult. The larger the size of the uterus, the greater the risk of significant blood loss and trauma to the surrounding structures. Uterine artery embolisation (UAE) has been in use since 1991 as a "noninvasive" treatment for uterine fibroids and has had encouraging results. Various workers have reported success rates between $86 \%$ and $100 \%$. We present here our experience with two patients who underwent embolisation of the uterine arteries, followed by hysterectomy. In both cases, patients had symptomatic uterine fibroids and no desire to conceive. The patients were counselled about the combined treatment and other treatment alternatives available to enable them make an informed choice.
\end{abstract}

Keywords Large uterine fibroids · Embolisation · Hysterectomy

\section{Introduction}

Hysterectomy for large uterine fibroids can prove to be a technically difficult procedure. The irregular shape of the uterus filling the pelvis may make access to the pedicles difficult. The larger the size of the uterus, the greater the risk of significant blood loss and trauma to the surrounding structures. Postoperative anaemia is associated with an increased incidence of infection in the wound and delayed general recovery. There are also risks associated with blood transfusion.

U. Khopkar $(\bowtie) \cdot$ R. M. Williams $\cdot$ P. Torrie

Department of Obstetrics and Gynaecology,

Royal Berkshire Hospital,

London Road, Reading,

Berkshire,

RG1 5AN, UK

E-mail: udeekay@yahoo.co.uk
Uterine artery embolisation (UAE) has been in use since 1991 as a "noninvasive" treatment for uterine fibroids and has had encouraging results. Various workers have reported success rates between $86 \%$ and $100 \%$ [1]. The procedure is safe and effective in appropriately trained hands. A combination of UAE with abdominal hysterectomy performed approximately $24 \mathrm{~h}$ later substantially reduces bleeding at operation, and the procedure becomes both safer for the patient and easier for the surgeon.

We present here our experience with two patients who underwent the combined technique. In both cases, patients had symptomatic uterine fibroids and no desire to conceive. The patients were counselled about the combined treatment and other treatment alternatives available.

The interventional radiologist, using a 5-French preshaped catheter, carried out embolisation using the Seldinger technique. The right femoral artery was catheterised, and the catheter was manipulated into the uterine artery. Once a stable position was confirmed on angiography, particulate material was infused until stasis was achieved in the artery, making sure there was no reflux into adjacent vessels. Materials used to occlude the vessel include polyvinyl alcohol (PVA), coils, embolospheres, or gel foam. Bilateral punctures are seldom required.

\section{Case 1}

CG, 56 years of age, para 0, presented with an abdominal swelling and pain. Clinical examination revealed a 22-weeks'-sized mass. On ultrasound examination, this was confirmed to be a uterine fibroid. Following discussion of treatment options, UAE followed by abdominal hysterectomy was agreed upon. The operation was performed $24 \mathrm{~h}$ after UAE. At hysterectomy, the uterus appeared to have shrunk and felt only 18 weeks in size, and it was remarkably pale and avascular. There was significantly reduced blood loss 
compared with similar cases in which UAE was not carried out prior to hysterectomy. The cut edges of the uterine vessels clearly showed the now gelatinous PVA particles. Preoperative haemoglobin was $13.6 \mathrm{~g} / \mathrm{dl}$ compared with $11.8 \mathrm{~g} / \mathrm{dl}$ on the $2 \mathrm{nd}$ postoperative day. The patient made an uneventful recovery with the usual postoperative care and thromboprophylaxis. She has since attended the outpatient clinic and is well. Histopathology confirmed the diagnosis of uterine fibroids. The uterus weighed $916 \mathrm{~g}$.

\section{Case 2}

DG, 44 years of age, para 0, presented with longstanding disabling menorrhagia leading to profound anaemia. This was corrected with red cell transfusions. The uterus was enlarged to 26 weeks' size, and there was a large fibroid protruding from the cervix into the vagina. She was also severely obese, with a body mass index of 44 . Hysterectomy was a definitive option for sustained recovery. She underwent UAE followed by a laparotomy $24 \mathrm{~h}$ later. The pelvic anatomy was severely distorted at operation. The fibroid arose from the anterior uterine wall, and the urinary bladder was adherent high up onto the fibroid. After the ovarian pedicles were secured and the bladder dissected, a myomectomy was performed. This removed not only the abdominal fibroid but also the vaginal portion of the fibroid. Hysterectomy was then completed in the usual way. Once again, there was minimal blood loss. Preoperative haemoglobin was $15 \mathrm{~g} / \mathrm{dl}$ and was $13.9 \mathrm{~g} / \mathrm{dl}$ on the 2 nd postoperative day. Histology confirmed the diagnosis.

\section{Discussion}

The role and efficacy of hysterectomy for symptomatic uterine fibroids is well established and noncontroversial. Hysterectomy is also associated with high patient satisfaction, with $85-90 \%$ of patients being satisfied with the operation and improved quality of life [2].

In this report, we have attempted to show that reduction in uterine vascularity prior to hysterectomy can help in facilitating the surgery, reducing operating time, and particularly, minimising blood loss at operation. Blood loss was assessed on the basis of the operative estimate and the difference between preoperative and postoperative haemoglobin levels.

Hillis et al. [3], in their study of uterine size and risk of complications among women undergoing abdominal hysterectomy for leiomyomas, found a significant increase in complications when uterine weight exceeded $500 \mathrm{~g}$, which corresponds to a uterine size of approximately 14-18 weeks. They also found that operative haemorrhage was more common among women with uterine weight greater than $500 \mathrm{~g}$. This may reflect greater perfusion associated with enlarged uterine leio- myomas. They also reported an increased incidence of complications such as cuff cellulitis, postoperative pyrexia, and pulmonary embolism.

Over the years, gynaecologists have attempted to reduce the size of the uterus prior to hysterectomy when the uterine size exceeded 14 weeks. Gonadotrophinreleasing analogue agonists ( $\mathrm{GnRHa}$ ) have been used for this purpose and to facilitate the vaginal approach for the same. Stovall et al. [4] concluded that the use of GnRHas reduces the need for preoperative blood transfusion and also facilitates a vaginal approach for surgery. However, they did not find a significant difference in patients whose uteri were more than 18 weeks prior to GnRHa treatment. Besides, GnRHas must be used for at least 2 months prior to surgery and are not without significant hypo-oestrogenic symptoms and cost.

Arterial embolisation has been used since the 1970s to control postpartum haemorrhage and to devascularise tumours and vascular malformations. In 1994, Ravina et al. [5] published their first series of 10 cases in which they carried out embolisation of the uterine arteries prior to myomectomy. The average blood loss was quoted to be just $54 \mathrm{ml}$, compared with between 400 and $800 \mathrm{ml}$ in similar cases without embolisation. Further, in 1995 Ravina et al. [6] published their series of 16 cases of $\mathrm{UAE}$ as the sole treatment for uterine myomas.

A study of perfusion and volume changes on magnetic resonance imaging was carried out by deSouza and Williams [7]. This study found that immediately following embolisation, perfusion was reduced dramatically in both the myometrium and the fibroid. However, at 1 and 4 months, myometrial perfusion returned to normal, whereas the fibroid perfusion remained suppressed. Immediate reduction in perfusion was invaluable in providing a relatively avascular field at operation in the two cases that we have reported.

There is only one other report in the literature in which this combined technique of UAE followed by definitive surgery has been used for treating a giant leiomyoma. Djelmis et al. [8] devascularised the giant uterine fibroid and then proceeded with the hysterectomy. The combined technique served the purpose by reducing operative blood loss, which in turn contributed to rapid postoperative recovery.

Both cases in our report took just over an hour for the surgical procedure. We are encouraged to offer this combined technique to other well-chosen patients who may benefit from it. The combined technique may be used in patients who object to receiving blood or blood products, such as Jehovah's Witnesses.

\section{References}

1. Royal College of Radiologists and Royal College of Obstetricians and Gynaecologists (2001) Clinical recommendations on the use of uterine artery embolisation in the management of fibroids. Report of a joint working party. RCOG Press, London 
2. Lumsden MA (2002) Embolisation versus myomectomy versus hysterectomy. Hum Reprod 17:253-259

3. Hillis S, Marchbanks PA, Peterson HB (1996) Uterine size and risk of complications among women undergoing abdominal hysterectomy for leiomyomas. Obstet Gynaecol 87:539-543

4. Stovall TG, Summit RL Jr, Washburn SA, Ling FW (1995) Gonadotropin releasing hormone agonist use before hysterectomy (comment). Am J Obstet Gynecol 172:1650-1651

5. Ravina JH, Merland JJ, Herbreteau D, Houdart E, Bouret JM, Madelenat P (1994) Embolisation pré-opératoire des fibromes utérins. Résultats préliminaries (10 cas). La Presse Médicale Octobre 23:1540
6. Ravina JH, Herbreteau D, Ciraru-Vigneron N, Bouret JM, Houdart E, Aymard A, et al. (1995) Arterial embolisation to treat uterine myomata. Lancet 346:671-672

7. deSouza NM, Williams AD (2002) Uterine arterial embolization for leiomyomas: perfusion and volume changes at MR imaging and relation to outcome. Radiology 222:367-374

8. Djelmis J, Mayer D, Majerovic M, Radanovic B, Starcevic V (2001) Giant uterine leiomyoma devascularized by embolization prior to surgical removal. Eur J Obstet Gynecol Reprod Biol 99:278-280 